This item was submitted to Loughborough's Research Repository by the author.

Items in Figshare are protected by copyright, with all rights reserved, unless otherwise indicated.

\title{
Planar revolute-coupled kinematic chains in critical configurations and their duals
}

PLEASE CITE THE PUBLISHED VERSION

http://dx.doi.org/10.1177/1464419313517114

PUBLISHER

(c) Sage

VERSION

AM (Accepted Manuscript)

\section{PUBLISHER STATEMENT}

This work is made available according to the conditions of the Creative Commons Attribution-NonCommercialNoDerivatives 4.0 International (CC BY-NC-ND 4.0) licence. Full details of this licence are available at: https://creativecommons.org/licenses/by-nc-nd/4.0/

\section{LICENCE}

CC BY-NC-ND 4.0

\section{REPOSITORY RECORD}

Davies, T.H., and L.P. Laus. 2019. "Planar Revolute-coupled Kinematic Chains in Critical Configurations and Their Duals”. figshare. https://hdl.handle.net/2134/16563. 


\title{
Planar Revolute-Coupled Kinematic Chains in Critical Configurations and their Duals
}

\author{
Trevor H. Davies* \\ Luís Paulo Laus ${ }^{\dagger}$
}

\begin{abstract}
The motivation for studies of dual coupling networks is explained and a definition is suggested for duality. Dual couplings are defined and several couplings dual with the revolute coupling are described. Six linkages, instantaneously in a critical configuration, provide examples. The consequences of these critical configurations are explored for both the linkages and the coupling networks that are dual with them.
\end{abstract}

Keywords: balance, graph, screw, stability, singularity, uncertainty

\section{Introduction}

This paper is concerned with simple planar kinematic chains coupled by revolute couplings (kinematic pairs) and also with coupling networks that are dual with these kinematic chains. The relative motions that can take place between bodies of the dual coupling networks attributable to underconstraint and the actions that can exist within them attributable to overconstraint are identical with the actions and motions, respectively, of the kinematic chains.

In particular, the paper is a study of characteristics of such kinematic chains and their duals when they are in a critical configuration. Such critical configurations are of two kinds. Kinematic chains in a critical configuration of the first kind are said to be in a stationary configuration. This term is used by Hunt [1] to describe a kinematic chain in motion configured in such a way that the relative motion of two directly coupled bodies is instantaneously zero. Gosselin and Angeles [2], refer to such configurations as singularities. (In [2], two different kinds of singularities are described, see Section 3.1 for details.)

It is more difficult to find such a succinct, yet selfexplanatory, label for critical configurations of the second kind. In this paper critical configurations of the second kind are called rank-reduced configurations because the rank of characteristic matrices decreases when such configurations are encountered. Again, other terms have been

\footnotetext{
*Wolfson School of Mechanical and Manufacturing Engineering, Loughborough University, Loughborough, Leicestershire LE11 3TU, UK, Email: T.H.Davies@lboro.ac.uk

${ }^{\dagger}$ Department of Mechanical Engineering (DAMEC), Federal University of Technology - Paraná (UTFPR), Av. 7 de Setembro, 3165, CEP: 80.230-901, Curitiba, PR, Brazil, Email: laus@utfpr.edu.br
}

used: Hunt [1] writes of uncertainty configurations, Gosselin and Angeles [2], of singularity of the third kind and Zlatanov et al. [3] of constraint singularity. A justification for the choice of the term rank-reduced is provided in Section 4, where relevant matrices are described in detail. Before any further discussion on the subject of critical configurations, we begin with the subject of duality.

Motivation for the work on duality culminating in this paper, and for earlier ones [4,5], has its origin in papers $[6,7]$ that establish four equations. These equations provide relationships between the magnitudes of the motions (first order kinematics) that pairs of bodies can experience attributable to underconstraint and between the magnitudes of actions that can exist attributable to overconstraint. Two of these four equations are adaptations of Kirchhoff's Laws.

$$
\begin{aligned}
& {\left[\hat{\mathbf{M}}_{N}\right]_{d l \times F} \boldsymbol{\psi}_{F}=\mathbf{0}_{d l}} \\
& {\left[\hat{\mathbf{A}}_{N}\right]_{d k \times C} \boldsymbol{\Psi}_{C}=\mathbf{0}_{d k}}
\end{aligned}
$$

Use of these equations requires some understanding of screw systems. Hunt [1] classifies screw systems and special screw systems. Screw systems relevant to this paper are discussed in greater detail in Section 3.1.3. The minimum order of the screw system to which all motion and action screws under consideration belong is the dimension $d(1 \leq d \leq 6) ; k$ and $l$ are the numbers of independent cutsets and circuits (loops); $C$ and $F$, the gross degrees of constraint (DOC) and freedom (DOF), are the sums of the DOC $c$ and DOF $f$ of all couplings. Matrices $\hat{\mathbf{M}}_{N}$ and $\hat{\mathbf{A}}_{N}$ contain the motion and action screw coordinates, respectively. The vectors $\boldsymbol{\psi}$ and $\boldsymbol{\Psi}$ contain the magnitudes of the motion and action screws, respectively.

An analysis of the dual coupling networks shown in Figure 1 is not provided because this example is topologically trivial, but an analysis of the dual coupling networks shown in Figure 5 is presented in Section 4.1.2.

One feature of publications $[8,9,10,11,12,13,14]$ that are concerned with duality in multi-body systems is the absence of a consensus about the meaning of duality and the means by which a dual can be found. In particular, a Stephenson Kinematic Chain is chosen as an example because we have disagreements with the coupling network, described as a static pillar system, that is claimed in [8] to be its dual. 


\section{Dual coupling networks}

Consider any coupling network $\mathrm{N}$ having matrices $\hat{\mathbf{M}}_{N}$ and $\hat{\mathbf{A}}_{N}$ in equations (1) and (2). The question arises as to whether there exists another coupling network $\mathrm{N}^{*}$, with matrices $\hat{\mathbf{M}}_{N}^{*}$ and $\hat{\mathbf{A}}_{N}^{*}$, for which $\hat{\mathbf{M}}_{N}^{*}=\hat{\mathbf{A}}_{N}$ and $\hat{\mathbf{A}}_{N}^{*}=$ $\hat{\mathbf{M}}_{N}$. If $\mathrm{N}^{*}$ does exist, $\mathrm{N}$ and $\mathrm{N}^{*}$ are called dual coupling networks and motions and actions that can exist in one are identical geometrically to actions and motions, respectively, in the other.

\subsection{A definition of duality}

Dual concepts can be physical or mathematical. For the purposes of the definition that follows let the two concepts be referred to as $\mathrm{A}$ and $\mathrm{A}^{*}$.

Duality exists between $\mathrm{A}$ and $\mathrm{A}^{*}$ if there is a set of sequential rules or instructions that, when applied to $\mathrm{A}$ creates $\mathrm{A}^{*}$, and when applied to $\mathrm{A}^{*}$, creates $\mathrm{A}$.

In $[4,5]$ two coupling networks $\mathrm{N}$ and $\mathrm{N}^{*}$ are described as dual if two conditions are met. These are that:

- the graphs of dual coupling networks, called coupling graphs, are dual graphs;

- and corresponding couplings of the dual coupling networks are dual couplings.

The expression "dual couplings" requires an explanation. A passive coupling can be characterised by two reciprocal screw systems [1] of order $f$ and $c$, where $f+c=6$. The $f$-system of motion screws is the system of minimal order $f$ to which all possible instantaneous motions allowed by the coupling belong. The $c$-system of action screws is the system of minimal order $c$ to which all possible actions that the coupling can transmit belong. Two couplings are referred to here, and in earlier papers [4, 5], as dual provided that the $f$-and $c$-systems of one are identical, geometrically, to the $c$ - and $f$-systems, respectively, of the other. Hereafter, in this paper, all features of the network $\mathrm{N}^{*}$ are identified by the use of an asterisk. To summarise, the rules for finding $\mathrm{N}^{*}$ the coupling network that is the dual of coupling network $\mathrm{N}$ are as follows.

1. Create the coupling graph $G_{C}$ of $\mathrm{N}$.

2. Create the graph $G_{C}^{*}$ that is the dual graph of $G_{C}$.

3. Intersecting edges of $G_{C}^{*}$ and $G_{C}$ represent dual couplings: thereby find the couplings of $\mathrm{N}^{*}$.

4. Create $\mathrm{N}^{*}$ by arranging for the $c^{*}$ - and $f^{*}$-systems of screws of each coupling of $\mathrm{N}^{*}$ to have instantaneous screw axes (ISA) with locations that are identical to those of the $f$ - and $c$-systems, respectively, of the corresponding couplings of $\mathrm{N}$.

Bruyninckx [11], writing about dual manipulators, distinguishes between topological, projective and metric duals. When $\mathrm{N}^{*}$ is assembled in accord with instruction 4 the coupling networks $\mathrm{N}$ and $\mathrm{N}^{*}$ are said to be metric duals.

\subsection{Formulae for the nett degrees of freedom and constraint}

Formulae for the nett DOF and DOC, $F_{N}$ and $C_{N}$, are derived [7] that each involve the rank $a$ of $\hat{\mathbf{A}}_{N}$ or the rank $m$ of $\hat{\mathbf{M}}_{N}$. It can be difficult to ascertain the rank of a matrix but the rank can be eliminated from two equations to provide the following equations

$$
\begin{aligned}
& F_{N}-C_{N}=F-d l \\
& C_{N}-F_{N}=C-d k .
\end{aligned}
$$

Often $F_{N}$ is easy to establish, inspection may be sufficient, whereas this is rare for $C_{N}$. Thus equations (3) and (4) provide two useful formulae for $C_{N}$.

\subsection{The dual of a revolute coupling}

A revolute coupling is characterised by a single motion screw of zero pitch, namely an angular velocity. The DOF $f$ and DOC $c$ are one and five, respectively. The dual coupling must be capable of transmitting a single action screw of zero pitch, namely a force. There are several couplings capable of doing this but some of them are imperfect as duals of the revolute coupling. This is because the angular velocity allowed by a revolute coupling can be a vector in either direction but, for some couplings that can only transmit a force, this force is unidirectional. A cord must remain in tension so that the force is unidirectional unless there are two collinear cords. Likewise, a single point contact between two bodies can only transmit a unidirectional force unless there are two such contacts sharing the same common normal. As well as these passive couplings there are active couplings [15] that are capable of transmitting unidirectional force; two examples are gravitational and magnetic couplings. Both are disregarded in this paper: all bodies are assumed to be weightless.

The coupling used hereafter in this paper where the dual of a revolute coupling is required is a compound coupling that comprises two spheric $(\mathrm{S})$ couplings in series. Such couplings are shown in the lower two drawings of Figure 1.

\section{Planar four-bar linkages in critical configurations and their duals}

In this section, a distinction is made between four-bar linkages instantaneously configured so that three of the four ISA are coplanar and those in which all four ISA are coplanar.

\subsection{Three coplanar ISA}

Two views of a planar four-bar linkage in a critical configuration are shown in the upper half of Figure 1. At the instant the linkage is in the position shown, points $\mathrm{A}, \mathrm{B}$ and $\mathrm{D}$ are collinear so that the length $\mathrm{BD}$ and the angle $\mathrm{BCD}$ are both minimum. Thus the relative angular velocity of links BC and CD is instantaneously zero. Such a linkage is said to be [1] in a stationary configuration and bearing $\mathrm{C}$ as 


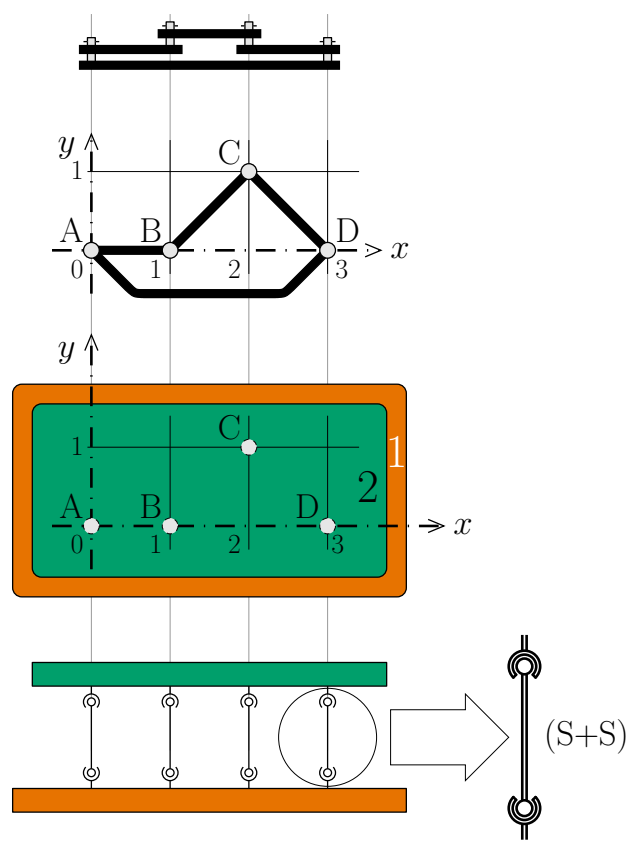

Figure 1: Two views of a planar four-bar linkage N (top) and of the coupling network $\mathrm{N}^{*}$ that is its dual (bottom)

being transitorily inactive. The circumstances illustrated in Figure 1 occur also when the ISA A, C and D are coplanar and the angular velocity of coupling $\mathrm{B}$ is transitorily zero.

Gosselin and Angeles [2] refer to these configurations as being singularities of the first or second kind. This distinction does not depend on which coupling is inactive but, instead, on which pairs of bodies are input and output ports. This paper is concerned with kinematic chains and their duals. It has been argued [15] that a kinematic chain only becomes a mechanism after input and output ports have been identified. So the distinction that has been made [2] between singularities of the first and second kind is not needed in this paper.

The four-bar linkage is referred to in this section as coupling network N. The coupling graph $G_{C}$ corresponding to network $\mathrm{N}$, and the graph $G_{C}^{*}$ dual with $G_{C}$, are both shown in Figure 2. The edges of $G_{C}$ are solid lines and the edges of $G_{C}^{*}$ are dashed lines. The orientation indicated by an arrow on each edge of these graphs records the arbitrarily chosen sign convention for motions and actions. Thus, the arrow on edge $\mathrm{A}$ in graph $G_{C}$ records the decision that a vector directed in the positive $z$-axis direction represents the angular velocity of link AB relative to link DA, not the converse. Because the coupling graph $G_{C}^{*}$ comprises two nodes and four edges each terminating at a different node, the dual coupling network $\mathrm{N}^{*}$ must comprise two bodies coupled by four couplings. Each of these four couplings must be a dual of a revolute coupling. Two views of $\mathrm{N}^{*}$ are shown in the lower half of Figure 1.

\subsubsection{The stabiliser coupling in $\mathrm{N}^{*}$}

In $\mathrm{N}$, the angular velocity of coupling $\mathrm{C}$ is instantaneously zero; dually, in $\mathrm{N}^{*}$, no force is transmitted by coupling $\mathrm{C}$. In the absence of coupling $\mathrm{C}$ the two bodies of $\mathrm{N}^{*}$ are in an

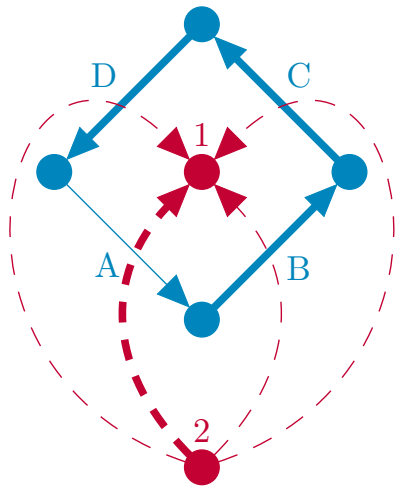

Figure 2: The dual coupling graphs $G_{C}$ (solid lines) and $G_{C}^{*}$ (dashed lines) of the dual coupling networks shown in Figure 1. Thick lines indicate the branches of a spanning tree and thin lines the chords in both $G_{C}$ and $G_{C}^{*}$

unstable configuration [16]; they are free to rotate relative to one another about the line ABD in either direction. To avoid this, the coupling $\mathrm{C}$ must be capable of transmitting force in either direction. Such a coupling is reminiscent of the extra wheel called a stabiliser that can be attached to a child's bicycle while the child is learning to ride. The term stabiliser coupling seems appropriate although, on the bicycle, the force on the stabiliser wheel is unidirectional.

\subsubsection{Freedom and constraint}

For the four-bar linkage $\mathrm{N}$ and its dual $\mathrm{N}^{*}, l$ and $k^{*}$ are both one, $F$ and $C^{*}$ are both four, and $F_{N}$ and $C_{N}^{*}$ are both one. Thus the magnitude of all four angular velocities in $\mathrm{N}$ and all four forces in $\mathrm{N}^{*}$ can be expressed in terms of any one of them that has a non-zero value. It can be recognised from Figures 1 and 2 that $k$ and $l^{*}$ are both three, and $C$ and $F^{*}$ are both 20 . Using equation 3, and $d=6, C_{N}$ and $F_{N}^{*}$ are found to both be three.

\subsubsection{Motions of bodies in the four-bar linkage $\mathrm{N}$ and actions within its dual, $\mathrm{N}^{*}$}

The first time derivative of displacements, called here by the shorthand term motions, can be represented geometrically as screws. This is true also for actions. The screw has an ISA, a pitch $p$ that is a length equal to the torque/force ratio for actions and translational velocity/angular velocity ratio for motions. Geometrically, the only difference between action and motion screws is the units used for the magnitude of these quantities. The motion screws of all pairs of bodies of a planar kinematic chain belong to a $5^{\text {th }}$ special 3 -system of screws. The screws of this $5^{\text {th }}$ special 3 -system are of two kinds. There are screws of the same finite pitch $p$ with parallel axes and screws of infinite pitch that are free vectors having any direction perpendicular to those with parallel axes. Another way of describing the screws of infinite pitch is by imagining screws of finite pitch with ISA at infinity but of infinitesimal magnitude. Furthermore, for planar kinematic chains, the pitch $p$ of the finite pitch screws is zero so the ISA are rotation axes. The action screw sys- 
tems to which all the forces transmitted by couplings of $\mathrm{N}^{*}$ belong is an identical $5^{\text {th }}$ special 3 -system of screws of zero pitch.

When these networks $\mathrm{N}$ and $\mathrm{N}^{*}$ are not in a critical configuration each of the four subsets of three screws belong to the same $5^{\text {th }}$ special 3 -system to which all four screws belong. But when they are in a critical configuration, as depicted in Figure 1, the subset of screws with axes through A, B and D belong to a $2^{\text {nd }}$ special 2 -system of screws. The ISA of this special 2-system are again lines parallel with the $z$-axis but now only the subset of those lines that pass through points of the $x$-axis.

\subsubsection{Actions within the four-bar linkage $\mathrm{N}$ and motions of bodies in its dual, $\mathrm{N}^{*}$}

The action screws that can exist within $\mathrm{N}$ and the motion screws of bodies in $\mathrm{N}^{*}$ again both belong to the $5^{\text {th }}$ special 3-system of screws. Accurate manufacture will ensure that any actions within $\mathrm{N}$ are of low magnitude. It is interesting to note that the actions within planar mechanisms that are attributable to active couplings [15] belong to another screw system, the $4^{\text {th }}$ special 3 -system of screws. These actions, mainly coplanar forces, are created by active couplings [12] that are not present in kinematic chains but are in machinery. In machinery these actions can coexist alongside actions of the $5^{\text {th }}$ special 3 -system of screws. There is one important difference between the relative rotations of pairs of bodies in $\mathrm{N}$ and $\mathrm{N}^{*}$. In $\mathrm{N}^{*}$ the rotation is transitory. Another way of describing this is to state that there is infinitesimal resistance provided within $\mathrm{N}^{*}$ to infinitesimal rotations. Resistance increases rapidly during a finite displacement because of work done that is stored as strain energy, mainly in extending and compressing elastic material within the $(\mathrm{S}+\mathrm{S})$ couplings.

When the linkage $\mathrm{N}$ is in the critical configuration shown in Figure 1 the action screws within $\mathrm{N}$ and motion screws allowed in $\mathrm{N}^{*}$ permitted by the screws of the subset $\mathrm{A}$, $\mathrm{B}$, and $\mathrm{D}$ contain all the screws of the $5^{\text {th }}$ special 3 -system previously described. In addition, the absence of the screw at $\mathrm{C}$ from this subset means that there is also a screw of zero pitch with an ISA coincident with the $x$-axis. Together, these screws belong to a $2^{\text {nd }}$ special 4-system of screws that contains screws reciprocal to the screws of the $2^{\text {nd }}$ special 2-system described in Section 3.1.3.

\subsection{A three-bar planar linkage with transitory freedom}

Suppose now that the bearing $\mathrm{C}$ is locked so that, in effect, members $\mathrm{BC}$ and $\mathrm{CD}$ can be replaced by a rigid triangle BCD as shown in Figure 3. The three remaining motion screws of $\mathrm{N}$ and action screws of $\mathrm{N}^{*}$ have coplanar and parallel ISA so they belong to the same $2^{\text {nd }}$ special 2 -system of screws. As a consequence the rank $a$ of matrix $\hat{\mathbf{A}}_{N}$ and the rank $m$ of matrix $\hat{\mathbf{M}}_{N}$ are reduced by one. It is for this reason that critical configurations of this second kind are called rank-reduced configurations. The reduction in the order of the screw system for the subset of screws associated with the actual system movement is akin to the appear-

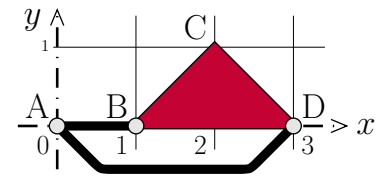

Figure 3: Three-bar planar linkage derived from Figure 1 with coplanar ISA

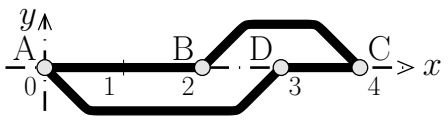

Figure 4: Four-bar planar linkage derived from Figure 1 with coplanar ISA

ance of empty columns in the matrix $\mathbf{J}_{C}$ created from the cofactors of the Jacobian of a manipulator [17, 18].

The gross DOF $F$ of $\mathrm{N}$ is reduced from four to three, however the nett DOF $F_{N}$ remains one but now only transitorily. Dually, $C_{N}^{*}$ is also one and the removal of the stabiliser coupling means that the three forces in $\mathrm{N}^{*}$ are critically balanced because, without the perfect alignment of the ISA, the magnitude of the forces would be zero. Using equation (3), $C_{N}=4$, and so, dually, $F_{N}^{*}=4$. The additional constraint in $\mathrm{N}$ and freedom in $\mathrm{N}^{*}$ is recognised as a zero pitch screw in the $z=0$ plane, a general 1-system, with its ISA along ABD. It is a force in $\mathrm{N}$ and an angular velocity in $\mathrm{N}^{*}$.

\subsection{Four parallel coplanar ISA}

Another rank-reduced configuration is shown in Figure 4. This is a four-bar kinematic chain with link lengths that satisfy the condition:

$$
\mathrm{AB}+\mathrm{BC}=\mathrm{AD}+\mathrm{DC} .
$$

The critical configuration arises when the four ISA are coplanar. Hunt [1] describes such a kinematic chain as being in an uncertainty configuration because there are two possible consequences of a small displacement. At this instant the rank of matrices $\hat{\mathbf{A}}_{N}$ and $\hat{\mathbf{M}}_{N}$ is reduced by one. Unlike the three-bar linkage of Figure 3, the forces within the dual $\mathrm{N}^{*}$ of the kinematic chain $\mathrm{N}$ of Figure 4 , are not critically balanced. This is because, for four-bar linkage of Figure $4, F_{N}$ and $C_{N}^{*}$ are both in transition from one and two, instead of zero and one. Gosselin and Angeles [2] refer to this configuration as a singularity of the third kind. Such linkages are illustrated and discussed in [3, 19, 20].

For linkages transitorily in this configuration, and the coupling networks that are dual with them, all four screws belong to a $2^{\text {nd }}$ special 2 -system of screws. $F_{N}$ and $C_{N}^{*}$ are both two, but $F_{N}$ is only transitorily two. After a small displacement $F_{N}$ reverts to one. Also, equation (3) shows that $C_{N}$ and $F_{N}^{*}$ are both four. The increase from three to four accounts for the possible existence in $\mathrm{N}$ of a force along the line $\mathrm{ABDC}$ and, in $\mathrm{N}^{*}$, of a possible rotation about this line. 


\section{The Stephenson Kinematic Chain and its dual}

Shai and Pennock [8] describe coupling networks called pillar systems and then seek their duals that are revolute coupled planar kinematic chains. These pillars, also called rods in [8], have some similarities with the $(\mathrm{S}+\mathrm{S})$ couplings shown in Figure 1. It is stated in [8] that the rods are connected to the plates by revolute joints. The $c$ and $f$ values for the dual of an $\mathrm{R}$ coupling must be one and five, respectively, whereas, for the $(\mathrm{R}+\mathrm{R})$ coupling, those values are four and two. Of the examples in [8] the one of particular interest is an example of a Stephenson Kinematic Chain, correctly labelled in Figure 9 of [8] but referred to as an eight-bar linkage in Figure 4 of [8].

\subsection{A Stephenson Kinematic Chain}

The Stephenson Kinematic Chain is referred to in this section as network N, and shown here in Figure 5 together with $\mathrm{N}^{*}$, the dual of N. Figure 6 shows the coupling graph $G_{C}$ of $\mathrm{N}$, using solid lines, and the coupling graph $G_{C}^{*}$ of $\mathrm{N}^{*}$, using dashed lines. These coupling graphs differ from the corresponding graphs shown in [8]. In addition to some labelling errors, in essence this difference arises because the ternary link shown coupled to other bodies by bearings at A, D and $\mathrm{F}$ in Figure 5, is regarded in [8] as three separate members. The consequence is that the coupling graph of the pillar system, called the PLGR (potential line graph representation), shown as Figure 5 in [8], contains vertices and edges representing additional bodies and couplings that enclose an extra region in the PLGR. The result is that the static pillar system contains four bodies, not the three of $\mathrm{N}^{*}$ shown here in Figure 5.

Like the four-bar linkage and its dual, the motion screws of $\mathrm{N}$ and $\mathrm{N}^{*}$ belong to a $5^{\text {th }}$ special 3 -system the action screws of $\mathrm{N}$ and $\mathrm{N}^{*}$, reciprocal with the motion screws, belongs to another $5^{\text {th }}$ special 3 -system. This Stephenson kinematic chain, like the four-bar of Figure 1, is in a stationary configuration; there is no reduction in the rank of matrices $\hat{\mathbf{A}}_{N}$ and $\hat{\mathbf{M}}_{N}$.

\subsubsection{Freedom and constraint}

For the Stephenson Kinematic Chain $\mathrm{N}$ and its dual $\mathrm{N}^{*}, l$ and $k^{*}$ are both two, $F$ and $C^{*}$ are both seven, and $F_{N}$ and $C_{N}^{*}$ are both one. It can be recognised from Figure 6 that $k$ and $l^{*}$ are both five, and $C$ and $F^{*}$ are both 35. Using equation (3), and $d=6, C_{N}$ and $F_{N}^{*}$ are found to both be six. The six independent freedoms within $\mathrm{N}^{*}$ are evident because now there are two bodies each of which can rotate independently, but transitorily, relative to the third. These motions, and the actions that can exist in $\mathrm{N}$, are described in greater detail in Section 4.2.

\subsubsection{Motion analysis of $\mathrm{N}$ and action analysis of $\mathrm{N}^{*}$}

Equations (1) and (2) can be used to find relationships between the magnitudes of motions and between the magnitudes of actions. Analyses of $\mathrm{N}$ and $\mathrm{N}^{*}$ can be carried out

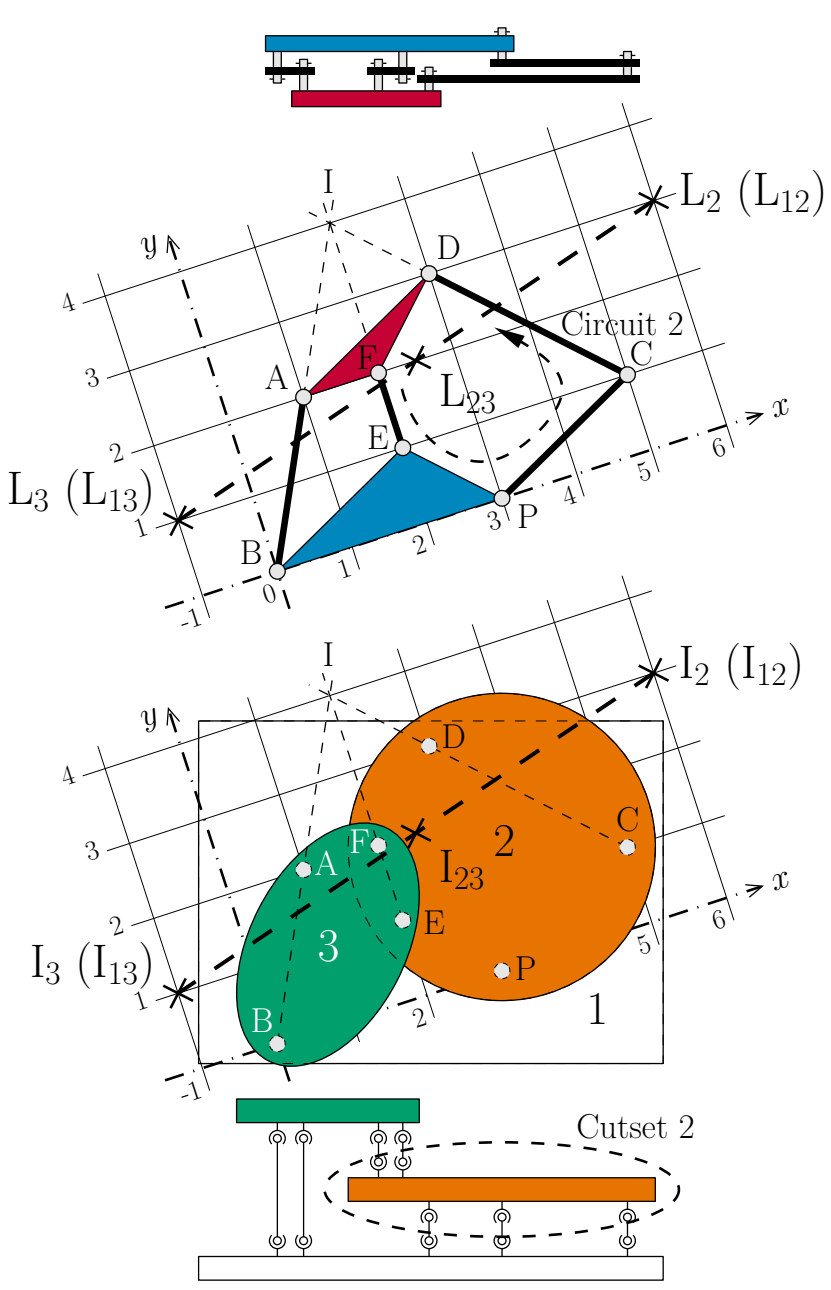

Figure 5: Two views of a Stephenson Kinematic Chain in a stationary configuration and of the coupling network that is its dual in an unstable configuration. Also, locations (L) of possible circuit forces in $\mathrm{N}$ and of cutset instantaneous rotation centres (I) in $\mathrm{N}^{*}$ are shown.

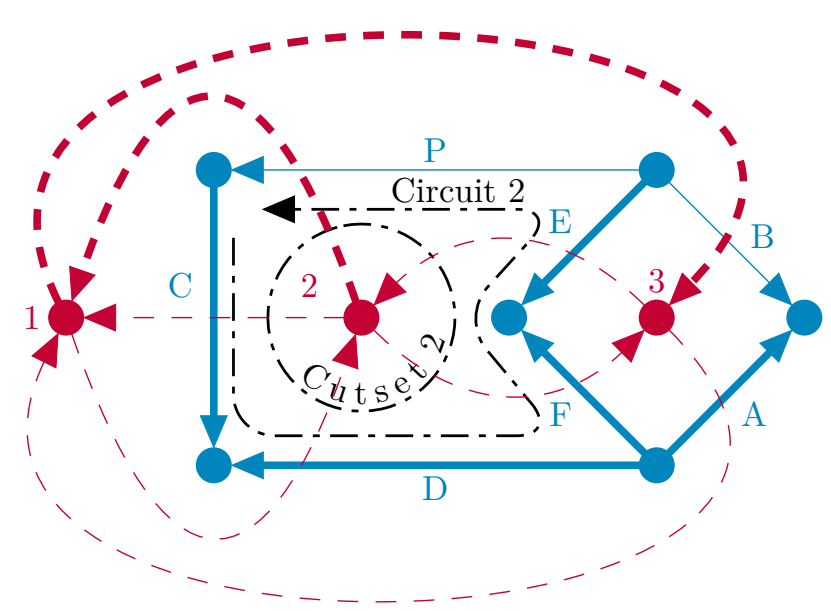

Figure 6: The dual coupling graphs $G_{C}$ (solid lines) and $G_{C}^{*}$ (dashed lines) of the dual coupling networks shown in Figure 5. Thick lines indicate the branches of a spanning tree and thin lines the chords of it in both $G_{C}$ and $G_{C}^{*}$ 
independently, but the effort involved is halved by arranging for matrices $\hat{\mathbf{A}}_{N}^{*}$ and $\hat{\mathbf{M}}_{N}^{*}$, the unit network action and motion matrices of $\mathrm{N}^{*}$, to be identical with matrices $\hat{\mathbf{M}}_{N}$ and $\hat{\mathbf{A}}_{N}$, respectively, of $\mathrm{N}$. These identities require the fundamental circuit matrix $\mathbf{B}$ of $G_{C}$ to be identical to the fundamental cutset matrix $\mathbf{Q}^{*}$ of $G_{C}^{*}$. The sign conventions, yet to be explained, used in labelling the dual coupling graphs $G_{C}$ and $G_{C}^{*}$ shown in Figure 6 ensures that matrices $\mathbf{B}$ and $\mathbf{Q}^{*}$ are identical. These matrices are:

$$
\begin{aligned}
& \begin{array}{lllllll}
A & B & C & D & E & F & P
\end{array} \\
& \mathbf{B}_{l \times e}=\mathbf{Q}_{k^{*} \times e}^{*}=\frac{\mathrm{B}}{\mathrm{P}}\left[\begin{array}{ccccccc}
-1 & 1 & 0 & 0 & -1 & 1 & 0 \\
0 & 0 & 1 & -1 & -1 & 1 & 1
\end{array}\right]
\end{aligned}
$$

where $e$ is the number of edges of $G_{C}$ and $G_{C}^{*}$. These two matrices are constructed by inspection of Figure 6 in respect to the spanning trees shown in thick lines both for $G_{C}$ and $G_{C}^{*}$. The choice of the spanning tree for $G_{C}$ is an arbitrary one, but, once this choice is made, the spanning tree of $G_{C}^{*}$ is determined. As Figure 6 shows, for the two matrices to be identical, the spanning tree of the dual graph $G_{C}^{*}$ is the unique spanning tree that ensures that the chords of one graph intersect the branches of the other. Furthermore, the positive senses assigned to circuits of $G_{C}$ and to cutsets of $G_{C}^{*}$ are such that they are consistent with the positive senses assigned to the corresponding chords of $\mathrm{N}$ and the branches of $\mathrm{N}^{*}$, respectively. In Figure 6 the positive senses assigned to edges of $G_{C}$, indicated by arrowheads on those edges, can be arbitrary decisions. For the matrices $\mathbf{B}$ and $\mathbf{Q}^{*}$ to be identical the positive senses assigned to the edges of $G_{C}^{*}$ must be those provided by turning the arrows of the corresponding edges of $G_{C}$ in a consistent direction, all clockwise or all counterclockwise. As Figure 6 shows, clockwise turns are used in this example.

Figure 6 also shows that $G_{C}$ has two chords labelled B and $\mathrm{P}$ and each has a corresponding fundamental circuit. Also, $G_{C}^{*}$ has two branches that can be labelled B and $\mathrm{P}$ and each has a corresponding fundamental cutset. The positive sense assigned to the two circuits of $G_{C}$ and the two cutsets of $G_{C}^{*}$ are such that, if a walk is taken in this positive direction along each circuit and cutset, the chords of $G_{C}$ and branches of $G_{C}^{*}$ are traversed in the positive sense that previously has been assigned to them.

The DOF of each coupling of $\mathrm{N}$ is equal to the DOC of the dual coupling of $\mathrm{N}^{*}$. A consequence is that, since $\mathbf{B}=\mathbf{Q}^{*}, \mathbf{B}_{M}=\mathbf{Q}_{A}^{*}$ also, where $\mathbf{B}_{M}$ is the fundamental circuit matrix of the motion graph $G_{M}$ of $\mathrm{N}$, and $\mathbf{Q}_{A}^{*}$ is the fundamental cutset matrix of the action graph $G_{A}^{*}$ of $\mathrm{N}^{*}$. Moreover, in this case the DOF of every coupling of $\mathrm{N}$ is one, therefore $F=C^{*}=e=e *$, and so

$$
\left[\mathbf{B}_{M}\right]_{l \times F}=\left[\mathbf{Q}_{A}^{*}\right]_{k^{*} \times C^{*}}=\mathbf{B}_{l \times e}=\mathbf{Q}_{k^{*} \times e}^{*} .
$$

Using the restricted dimension $d=3$ for screws of the $5^{\text {th }}$ special 3-system it follows that:

$$
\begin{aligned}
& {\left[\hat{\mathbf{M}}_{D}\right]_{d \times F}=\left[\hat{\mathbf{A}}_{D}^{*}\right]_{d \times C^{*}}=} \\
& t \text { and } W^{*}\left[\begin{array}{ccccccc}
\mathrm{A} & \mathrm{B} & \mathrm{C} & \mathrm{D} & \mathrm{E} & \mathrm{F} & \mathrm{P} \\
1 & 1 & 1 & 1 & 1 & 1 & 1 \\
2 & 0 & 1 & 3 & 1 & 2 & 0 \\
v \text { and } R^{*} & S^{*} \\
-1 & 0 & -5 & -3 & -2 & -2 & -3
\end{array}\right]
\end{aligned}
$$

where every column represents a motion screw $\{t ; u, v\}$ allowed in $\mathrm{N}$ and an action screw $\left\{W^{*} ; R^{*}, S^{*}\right\}$ transmitted in $\mathrm{N}^{*}$. The matrix $\hat{\mathbf{M}}_{N}$ is assembled from matrices $\hat{\mathbf{M}}_{D}$ and $\mathbf{B}_{M}$ as:

$$
\left[\hat{\mathbf{M}}_{N}\right]_{d l \times F}=\left[\begin{array}{c}
{\left[\hat{\mathbf{M}}_{D}\right]_{d \times F}{ }^{\left[\mathbf{B}_{1}\right]_{F \times F}}} \\
{\left[\hat{\mathbf{M}}_{D}\right]_{d \times F}\left[\mathbf{B}_{2}\right]_{F \times F}} \\
\vdots \\
{\left[\hat{\mathbf{M}}_{D}\right]_{d \times F}\left[\mathbf{B}_{l}\right]_{F \times F}}
\end{array}\right]_{d l \times F}
$$

where $\left[\mathbf{B}_{i}\right]_{F \times F}=\operatorname{diag}\left(\left[\mathbf{B}_{M}\right]_{i}\right)$ are diagonal matrices $(i=1,2, \ldots, l)$. The diagonal elements of $\left[\mathbf{B}_{i}\right]_{F \times F}$ are those of row $i$ of $\left[\mathbf{B}_{M}\right]_{l \times F}$. The matrix $\hat{\mathbf{A}}_{N}^{*}$ could be assembled in the same way from matrices $\hat{\mathbf{A}}_{D}^{*}$ and $\mathbf{Q}_{A}^{*}$ but, because of the identities, the resulting matrix is the same as $\hat{\mathbf{M}}_{N}$. Since $\mathbf{B}_{M}$ is equal to $\mathbf{B}$, see equation (6), the application of the matrices of equations (5) and (7) on equation (8) results in:

$$
\begin{aligned}
& {\left[\hat{\mathbf{M}}_{N}\right]_{d l \times F}=\left[\hat{\mathbf{A}}_{N}^{*}\right]_{d k^{*} \times C^{*}}=} \\
& \begin{array}{llllllll}
A & B & C & D & E & F & P
\end{array}
\end{aligned}
$$

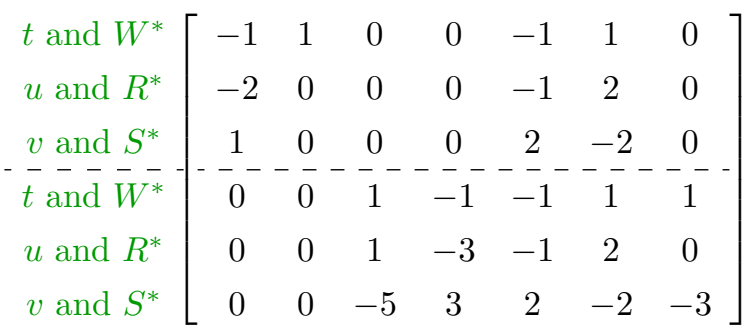

and these matrices have full rank, $m=a^{*}=6$. The upper three rows of equation (9) relate to circuit one of $\mathrm{N}$ and cutset one of $\mathrm{N}^{*}$; the lower three rows relate to circuit and cutset two.

The solutions,

$$
\boldsymbol{\psi}_{F}=\left[\begin{array}{lllllll}
t_{A} & t_{B} & t_{C} & t_{D} & t_{E} & t_{F} & t_{P}
\end{array}\right]^{T}
$$

for $\mathrm{N}$ and

$$
\boldsymbol{\Psi}_{C^{*}}^{*}=\left[\begin{array}{lllllll}
W_{A}^{*} & W_{B}^{*} & W_{C}^{*} & W_{D}^{*} & W_{E}^{*} & W_{F}^{*} & W_{P}^{*}
\end{array}\right]^{T}
$$

for $\mathrm{N}^{*}$ of equations (1) and (2), respectively, are both proportional to

$$
\left.\begin{array}{ccccccc}
\mathrm{A} & \mathrm{B} & \mathrm{C} & \mathrm{D} & \mathrm{E} & \mathrm{F} & \mathrm{P} \\
4 & 2 & 1 & 3 & 4 & 6 & 0
\end{array}\right]^{T}
$$

which is one possible basis for the null space of the matrix in equation (9). Vector (10) shows that, because $t_{P}=W_{P}^{*}=$ $0, \mathrm{~N}$ is in a stationary configuration and $\mathrm{N}^{*}$ is in an unstable configuration for reasons explained for the four-bar linkage of Figure 1.

Earlier it is explained that a motivation for duality studies is to seek the coupling network $\mathrm{N}^{*}$, dual with $\mathrm{N}$, for which the matrix $\hat{\mathbf{M}}_{N}^{*}=\hat{\mathbf{A}}_{N}$, and $\hat{\mathbf{A}}_{N}^{*}=\hat{\mathbf{M}}_{N}$. If the 
procedure for assigning positive senses to the edges and cutsets of $G_{C}^{*}$, described above, are not followed so that $\mathbf{Q}^{*} \neq \mathbf{B}$, and $\mathbf{B}^{*} \neq \mathbf{Q}$, then the result is that $\hat{\mathbf{M}}_{N}^{*} \neq \hat{\mathbf{A}}_{N}$, and $\hat{\mathbf{A}}_{N}^{*} \neq \hat{\mathbf{M}}_{N}$. Nevertheless, whatever the positive senses assigned to the edges and cutsets of $G_{C}^{*}$, the results shown in equations (9) and (10) remain valid.

The foregoing analysis of the Stephenson Kinematic Chain using equation (1) has also been completed for the other five examples described in this paper. Additionally, all six examples have been analysed using equation (2) to reveal actions in $\mathrm{N}$ and motions in $\mathrm{N}^{*}$. Details can be obtained from the second named author. In Section 6, Table 2 records the ranks $a$ and $m$ of matrices required by these analyses. As observed previously [7], for kinematic chains rank $a$ is invariably greater than rank $m$. For the Stephenson Kinematic Chain, rank $m$ of matrix $\hat{\mathbf{M}}_{N}$ in equation (9) is 6 whereas rank $a$ of $\hat{\mathbf{A}}_{N}$ is 29 .

\subsection{Spanning sets of screws and reciprocity}

In this section, the motions that can take place in $\mathrm{N}^{*}$ and actions transmitted in $\mathrm{N}$ are described.

\subsubsection{Spanning sets of screws in $\mathrm{N}$ and $\mathrm{N}^{*}$}

It is explained in Section 3.1.3 that the motions and actions screws of planar kinematic chains are zero pitch screws belonging to a $5^{\text {th }}$ special 3 -system of screws. All motions belonging to this screw system can be spanned by a set of three independent motions. One such set comprises an angular velocity $t$ about the $z$-axis and translational velocities $u$ and $v$ in the directions of the $x$ - and $y$-axes, respectively. It follows that the angular velocity components $r$ and $s$ about the $x$ - and y-axes are zero, as is translational velocity $w$ in the direction of the $z$-axis.

Likewise, actions can be spanned by three independent actions. One such set comprises the moments $R$ and $S$ of torque about the $x$ - and $y$-axes, respectively, together with a force $W$ along the $z$-axis. It follows that the moment of torque $T$ about the $z$-axis and forces $U$ and $V$ along the $x$ and $y$-axes, respectively, are each zero.

The two foregoing paragraphs can be repeated for the actions and motions of $\mathrm{N}^{*}$ by the addition of asterisks.

\subsubsection{Reciprocity}

From the results above it is noted that:

$$
r=s=T=U=V=w=0
$$

A consequence is that

$$
r R+s S+t T+u U+v V+w W=0 .
$$

Equation (11) demonstrates the claim [6, 7] that the notation is easier to remember than any alternative that has been proposed. Equation (11) also shows that no power is expended because the actions and motions are reciprocal to one another. It is this that allows the actions to exist in networks wherein motions can occur. Again, equation (11) can be repeated for $\mathrm{N}^{*}$ by adding asterisks to all terms.
Table 1: Circuits and cutsets in Figure 5

\begin{tabular}{cc}
\hline Circuit and cutset numbers & Set of couplings \\
\hline 1 & $\{\mathrm{~A}, \mathrm{~B}, \mathrm{C}, \mathrm{D}, \mathrm{P}\}$ \\
2 & $\{\mathrm{C}, \mathrm{D}, \mathrm{E}, \mathrm{F}, \mathrm{P}\}$ \\
3 & $\{\mathrm{~A}, \mathrm{~B}, \mathrm{E}, \mathrm{F}\}$ \\
\hline
\end{tabular}

\subsection{An example of circuit actions in $\mathrm{N}$ and cutset motions in $\mathrm{N}^{*}$}

In this section, an example is provided that shows the geometrical relationship between lines of actions of forces in $\mathrm{N}$ and between rotation axes in $\mathrm{N}^{*}$.

Because $F_{N}$ and $C_{N}^{*}$ are both one, the locations of axes of rotation within $\mathrm{N}$ and of the lines of action of forces within $\mathrm{N}^{*}$ are already determined and the only unknowns are the magnitude of one angular velocity in $\mathrm{N}$ and the magnitude of one force in $\mathrm{N}^{*}$. This is not so for forces in $\mathrm{N}$ and motions in $\mathrm{N}^{*}$. For these, arbitrary decisions must be made in order to provide an example. There are $\infty^{2}$ pairs of coordinates to select from when choosing the location of the point in the $z=0$ plane through which the ISA passes. For metric (i.e. perfect) duality, this ISA is both a line of action of a force in $\mathrm{N}$ and a rotation axis in $\mathrm{N}^{*}$.

The opportunity is taken here to describe actions and motions in terms of circuit actions and cutset motions, respectively. Because there are always fewer circuits and cutsets than edges in a graph it is well established in electrical circuit theory that circuit (loop or mesh) currents and cutset potential differences express information about electrical currents and potential differences more concisely than element currents and potential differences.

Electrical currents and actions are both through variables; electrical potential differences and motions are across variables [21]. Thus circuit actions most concisely describe actions that can exist within mechanical coupling networks and cutset motions most concisely describe relative motions of bodies belonging to those networks.

Table 1 lists and numbers three sets of couplings. The number of the set is the number of the body in $\mathrm{N}^{*}$ that would be isolated by the removal of the set of couplings of the same number for this example. Any two of the three sets can be chosen as independent or fundamental sets; the third set is dependent on the other two.

For this example the motions of bodies within $\mathrm{N}^{*}$ are described first. Cutset 2 is arbitrarily chosen as one of the two fundamental cutsets and the point with coordinates $(6,3)$ is arbitrarily chosen as the instantaneous centre of rotation of body 2 relative to bodies 1 and 3 . This point, labelled $\mathrm{I}_{2}$ in the lower diagram showing $\mathrm{N}^{*}$ in Figure 5, could be labelled $\mathrm{I}_{12}$ or $\mathrm{I}_{23}$. Instead, the subscript 2 is used to associate the motion with cutset 2 of couplings because, collectively, the couplings of cutset 2 allow the motion. To ensure metric duality, the same point $(6,3)$, labelled $\mathrm{L}_{2}$ in the diagram showing $\mathrm{N}$ in the upper half of Figure 5, represents the circuit action of circuit 2 in N. This is the force that could be transmitted by the couplings of circuit 2 that do not also belong to another fundamental circuit of couplings. Note that the location of $\mathrm{L}_{2}$ implies that each 
revolute coupling in circuit 2 must transmit force $\mathrm{L}_{2}$ and must also transmit the moment of $\mathrm{L}_{2}$ about the location of the coupling.

Next, two more arbitrary choices are made. First, cutset 3 is chosen as the second fundamental cutset. Second, the point $(-1,1)$ is chosen as the location of the instantaneous centre $\mathrm{I}_{3}$ for cutset 3 in the diagram of $\mathrm{N}^{*}$ and as the location of the force $\mathrm{L}_{3}$ for circuit 3 in the diagram of $\mathrm{N}$. Now $\mathrm{I}_{2}$ is also $\mathrm{I}_{12}$ but no longer $\mathrm{I}_{23}$ as well, and $\mathrm{I}_{3}$ is also $\mathrm{I}_{13}$.

In $\mathrm{N}$ the couplings $\{\mathrm{E}, \mathrm{F}\}$ belong to both circuit 2 and circuit 3 . So, the force transmitted by couplings $\{\mathrm{E}, \mathrm{F}\}$ is the resultant of the circuit forces of circuits 2 and 3 . Dually, in $\mathrm{N}^{*}$, the couplings $\{\mathrm{E}, \mathrm{F}\}$ belong to cutsets 2 and 3 . So, the angular velocity of bodies 2 and 3 allowed by couplings $\{\mathrm{E}, \mathrm{F}\}$ is the resultant of the cutset motions of cutsets 2 and 3. Because the dual coupling graphs $G_{C}$ and $G_{C}^{*}$ are directed graphs, they provide a record of an arbitrarily chosen sign convention for actions or motions. As explained in Section 4.1.2, the positive senses assigned to circuits 2 and 3 of $G_{C}$ are chosen to be consistent with the positive senses assigned to their associated chords, $\mathrm{P}$ and $\mathrm{B}$, respectively. A consequence of the direction assigned to edge $\mathrm{E}$ is that the action transmitted by coupling $\mathrm{E}$ is regarded as being exerted by the ternary link BEP on the binary link EF. Because the positive sense assigned to edge $\mathrm{E}$ is opposed to the positive senses assigned to circuits $\mathrm{P}$ and $\mathrm{B}$ of which edge $\mathrm{E}$ is a member, the force through $\mathrm{L}_{23}$, which is the resultant of the circuit actions in chords $\mathrm{P}$ and $\mathrm{B}$, is equal and opposite to the action transmitted by coupling E. Similar statements can be made about the action transmitted by coupling $\mathrm{F}$ which, in order to maintain the equilibrium of the binary link EF, must be equal and opposite to the force transmitted by coupling E. Similar statements can also be made about the equal and opposite relative (transitory) motions of bodies 2 and 3 about $\mathrm{I}_{23}$ allowed by couplings $\mathrm{E}$ and $\mathrm{F}$ of network $\mathrm{N}^{*}$.

Geometrically, the resultant of two zero-pitch screws belonging to the $5^{\text {th }}$ special 3 -system of screws is easily found. For these screws there is the theorem that, in its general form, may be described as the theorem of three parallel ISA. The theorem states that the three ISA are coplanar and the location of one screw relative to the two others is determined by the ratio of the magnitudes of the other two. The theorem has dual forms.

For N, the theorem requires that the resultant of circuit forces for circuits 2 and 3 has a line of action that passes through a point $\mathrm{L}_{23}$ on the line joining $\mathrm{L}_{2}$ and $\mathrm{L}_{3}$ and the location of $\mathrm{L}_{23}$ on that line is determined by the ratio of the magnitudes of the two circuit forces. This resultant is the force transmitted by couplings $\mathrm{E}$ and $\mathrm{F}$ of network $\mathrm{N}$ because these couplings belong to both circuits.

The circuit 2 of $\mathrm{N}$ and cutset 2 of $\mathrm{N}^{*}$ in Figure 5 have a dual relationship that appears clearly in Figure 6: both are drawn around node 2 . In $G_{C}$, the positive sense (counter clockwise) assigned to circuit 2 corresponds with the positive sense assigned to the associated chord P. In $G_{C}^{*}$, the positive sense (away from node 2 ) assigned to cutset 2 corresponds with the positive sense assigned to the branch $\mathrm{P}$. The circuit or cutset positive sense is used together with the edge sense, respectively of $G_{C}$ or $G_{C}^{*}$, to write the equation (5).

For angular velocities with parallel axes the theorem is better known as the theorem of three centres. This theorem, applied to $\mathrm{N}^{*}$, states that the resultant of cutset angular velocities for cutsets 2 and 3 has a rotation axis that passes through a point $\mathrm{I}_{23}$ on the line joining $\mathrm{I}_{2}$ and $\mathrm{I}_{3}$ and the location of $\mathrm{I}_{23}$ on that line is determined by the ratio of the magnitudes of the two cutset angular velocities. This resultant is the angular velocity allowed by couplings $\mathrm{E}$ and $\mathrm{F}$ of network $\mathrm{N}^{*}$ because these couplings belong to both cutsets.

\section{Variations on the theme of the Stephenson Kinematic Chain}

In this section, two modified forms of the Stephenson Kinematic Chain are described. Both are rank-reduced configurations.

\subsection{Alignment of points $D, C$, and $P$}

If bodies BEP and CP in Figure 5 are modified such that coupling $\mathrm{P}$ is relocated to a point on the dashed line through CD, to $(6,0)$ as shown in Figure 7 for example, the Stephenson Kinematic Chain will be in a rank-reduced configuration analogous to the four-bar linkage. For this configuration, $F_{N}$ and $C_{N}^{*}$ increase from one to two, and $C_{N}$ and $F_{N}^{*}$ from six to seven.

\subsubsection{Screw systems}

Consider the motions that are possible within $\mathrm{N}^{*}$. In addition to motions belonging to the $5^{\text {th }}$ special 3 -system, there are extra ones that cannot occur for bodies of $\mathrm{N}^{*}$ shown in Figure 5, but they can for bodies of $\mathrm{N}^{*}$ shown in Figures 7 and 8 . These extra motions are rotations about the three dashed lines. The three rotations are dual with the forces that can exist in links $\mathrm{AB}, \mathrm{EF}$ and $\mathrm{DC}$ in network $\mathrm{N}$ that cannot be present in the corresponding links of $\mathrm{N}$ in Figure 5 . These three screws of zero pitch belong to (and therefore define) a $1^{\text {st }}$ special 2 -system of screws. The system comprises $\infty^{1}$ screws of the same pitch, zero pitch in this example, with ISA that form a pencil of lines in the plane $z=0$ and with a centre located at I, the point of intersection of the three dashed lines located at $(2,4)$. If the magnitude of any one of the three angular velocities/forces in $N^{*} / N$ is known then the magnitude of the other two is easily found, for example, graphically, using a triangle of planar angular velocity/force vectors.

We seek the union of the 3- and 2-systems of screws. For a screw system to be a 5 -system of screws it must be possible to identify five linearly independent screws, i.e. screws that do not all belong to the same 4-system in its general or any of its five special forms [1]. Five such screws of zero pitch are easily identified in Figures 7 and 8. There are three screws of the 3 -system with ISA parallel with the $z$-axis through any three non-collinear points among the $\infty^{2}$ points in the plane $z=0$. There are also two screws with ISA that are 


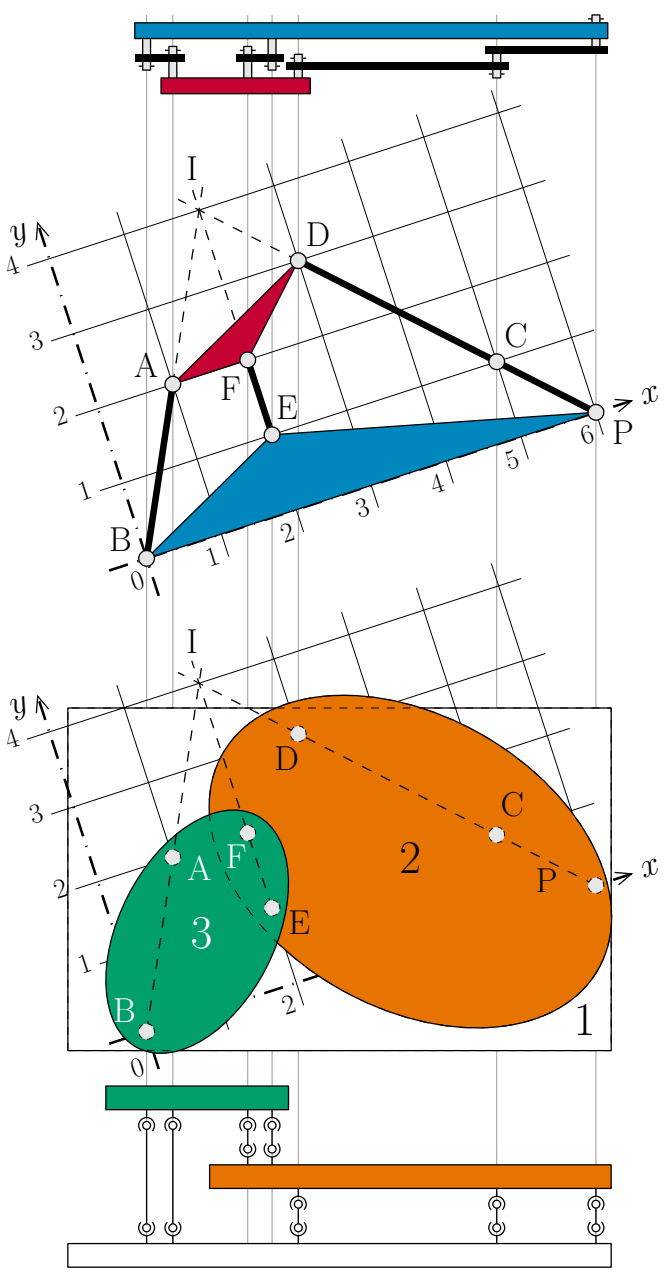

Figure 7: Modification of Figure 5 resulting in the alignment of points $\mathrm{D}, \mathrm{C}$, and $\mathrm{P}$

any two of the $\infty^{1}$ lines that belong to the pencil of lines through I that lie in the plane $z=0$.

Furthermore, reciprocal to a 5 -system is a 1 -system. This comprises a single screw and it is clear that, for the 5 -system that has been described, the 1-system comprises the single screw of zero pitch with an ISA through point I parallel with the $z$-axis. Because the pitch is finite it is a general 1system, not a special 1-system. It follows that the 5-system is also a general 5-system. In $\mathrm{N}^{*}$, consider a force with a line of action along the ISA through I parallel with the $z$ axis. This force cannot do work on any of the five linearly independent motion screws of $\mathrm{N}^{*}$ that have been described, and so it is reciprocal to them. Likewise, none of the $\infty^{5}$ possible actions screws of all magnitudes and pitches that can exist in $\mathrm{N}$ can do work on a rotation about the screw of zero pitch through I.

\subsection{A five-bar planar linkage with transitory freedom}

Suppose the link CP is removed and the shape of triangle BEP is again changed to become link BEC coupled directly to the binary link $\mathrm{CD}$ by revolute coupling $\mathrm{C}$ at $(5,1)$ as shown in Figure 8. A five-bar linkage is thereby created that is another example of a rank-reduced configuration similar to the three-bar linkage of Figure 3. Were it

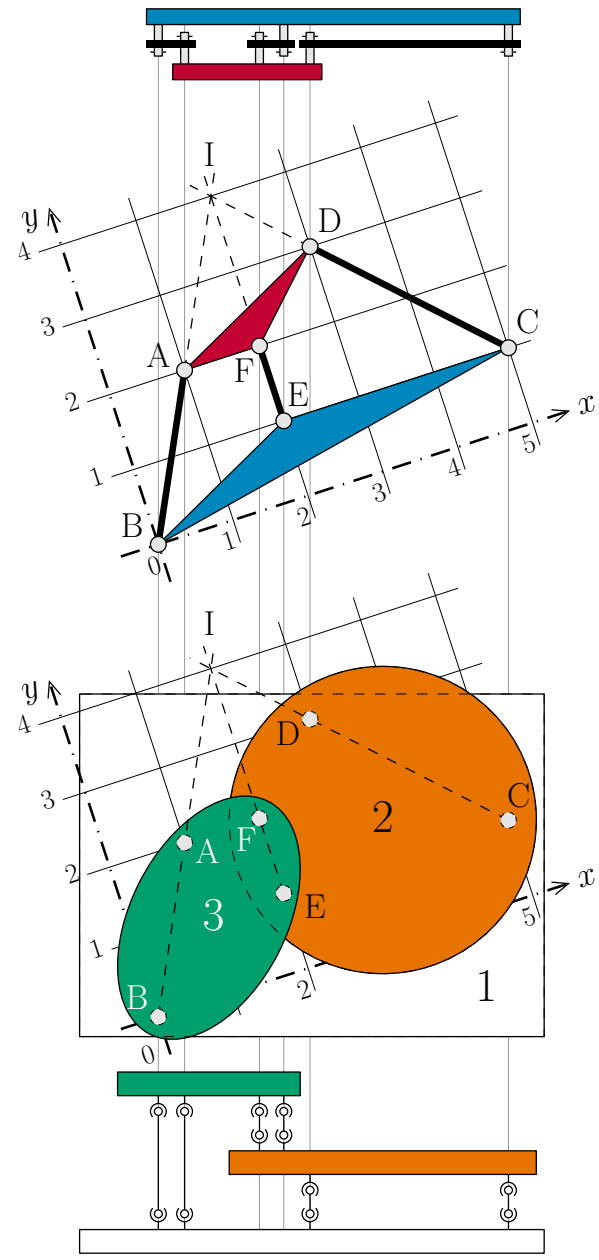

Figure 8: Modification of Figure 5 resulting in a five-bar linkage

not for the concurrence of the three dashed lines shown in Figure 8 the linkage would be a simply stiff planar truss. Furthermore, because $F_{N}$ is zero for a truss $\mathrm{N}$, dually $C_{N}^{*}$ would also be zero for its dual $\mathrm{N}^{*}$, implying that no set of forces could be in equilibrium so that no forces could exist. The absence of coupling $\mathrm{P}$ means that the gross DOF $F$ is reduced from seven to six but, because of the concurrence of lines at $(2,4)$, the nett DOF $F_{N}$ remains one, albeit transitorily. Dually, $C_{N}^{*}$ is one but, with no stabiliser coupling, the six forces through the $(\mathrm{S}+\mathrm{S})$ couplings are critically balanced because, if the six ISA are not located along the three concurrent lines, no forces can exist. Equation (3) shows that $C_{N}$ and $F_{N}^{*}$ remain seven and the additional constraint within $\mathrm{N}$, and freedom within $\mathrm{N}^{*}$, means that, like the linkage shown in Figure 7 , the three screws with ISA along the dashed lines belong to a $1^{\text {st }}$ special 2 -system of screws. No subset of motion/action screws of $\mathrm{N} / \mathrm{N}^{*}$ are linear dependent, but all the six screws are linear dependent and, consequently, $m=a^{*}=5$ and not 6 ; so this example is in a rank-reduced configuration.

\section{Conclusions}

The paper is a report on studies of planar revolute-coupled kinematic chains in critical configurations and of the cou- 
Table 2: A summary of some characteristics of dual coupling networks illustrated in this paper

\begin{tabular}{|c|c|c|c|c|c|c|c|c|}
\hline \multirow[t]{2}{*}{ Figure } & \multicolumn{2}{|c|}{$\begin{array}{c}\text { Ranks } m, a \\
\text { of } \hat{\mathbf{M}}_{N} \text { and } \hat{\mathbf{A}}_{N}\end{array}$} & \multicolumn{2}{|c|}{$\begin{array}{c}\text { Number of } \\
\text { circuits } l \text { and } \\
\text { cutsets } k\end{array}$} & \multicolumn{2}{|c|}{$\begin{array}{c}\text { Gross degree of } \\
\text { freedom } F \text { and } \\
\text { constraint } C\end{array}$} & \multicolumn{2}{|c|}{$\begin{array}{l}\text { Nett degree of } \\
\text { freedom } F_{N} \text { and } \\
\text { constraint } C_{N}\end{array}$} \\
\hline & $m=a^{*}$ & $a=m^{*}$ & $l=k^{*}$ & $k=l^{*}$ & $F=C^{*}$ & $C=F^{*}$ & $F_{N}=C_{N}^{*}$ & $C_{N}=F_{N}^{*}$ \\
\hline $1^{\mathrm{A}}$ & 3 & 17 & 1 & 3 & 4 & 20 & 1 & 3 \\
\hline $3^{\mathrm{B}}$ & 2 & 11 & 1 & 2 & 3 & 15 & 1 & 4 \\
\hline $4^{\mathrm{B}}$ & 2 & 16 & 1 & 3 & 4 & 20 & 2 & 4 \\
\hline $5^{\mathrm{A}}$ & 6 & 29 & 2 & 5 & 7 & 35 & 1 & 6 \\
\hline $7^{\mathrm{B}}$ & 5 & 28 & 2 & 5 & 7 & 35 & 2 & 7 \\
\hline $8^{\mathrm{B}}$ & 5 & 23 & 2 & 4 & 6 & 30 & 1 & 7 \\
\hline
\end{tabular}

Notes: ${ }^{\mathrm{A}} \mathrm{N}$ is in a stationary configuration, $\mathrm{N}^{*}$ is in an unstable configuration;

$\mathrm{B}$ both $\mathrm{N}$ and $\mathrm{N}^{*}$ are in a rank-reduced configuration

pling networks that are their duals. A definition of duality is suggested, dual graphs and dual couplings are explained, a coupling that is an ideal dual of the revolute coupling is identified and the procedure for finding the dual $\mathrm{N}^{*}$ of any coupling network $\mathrm{N}$ is provided.

Two kinds of critical configurations are identified. A kinematic chain $\mathrm{N}$ that is in a critical configuration of the first kind is described as being in a stationary configuration, the dual $\mathrm{N}^{*}$ is said to be in an unstable configuration. Within a kinematic chain $\mathrm{N}$ in a stationary configuration there is a coupling that is transitorily inactive. The dual coupling in $\mathrm{N}^{*}$ does not transmit an action; the term stabiliser coupling is introduced for this coupling. A characteristic of critical configurations of this first kind is that there are zero elements in the null spaces of the network motion matrix of $\mathrm{N}$ and the network action matrix of $\mathrm{N}^{*}$.

A kinematic chain $\mathrm{N}$ and its dual $\mathrm{N}^{*}$ that are in a critical configuration of the second kind are both described as rankreduced configurations in this paper because the rank of the network action and motion matrices falls by one from their normal values. For $\mathrm{N}$ and $\mathrm{N}^{*}$ there is a reduction, transitorily, in the order of the screw systems to which actions and motions belong. This is accompanied by an increase in the nett degrees of freedom and constraint.

All six examples described have been analysed using equations (1) and (2) and some results are recorded in Table 2. The only analyses recorded in full in this paper are those for a Stephenson Kinematic Chain using equation (1) and its dual using equation (2).

One reason for this choice is to correct a study conducted by others. For the Stephenson Kinematic Chain N and its dual $\mathrm{N}^{*}$, the opportunity is taken to describe actions within $\mathrm{N}$ in terms of circuit actions and to describe the motions of bodies within $\mathrm{N}^{*}$ in terms of cutset motions. Be aware that, the economies resulting from recording actions and motions in this way only become apparent for more complex networks than those described here. Studies of the kind described in this paper provide tutorial material in graph theory, screw theory and duality. Knowledge of these topics improves understanding of dangers that can arise, such as unexpected failure caused by overconstraint.

\section{Acknowledgement}

This work was partially supported by two Brazilian national foundations: $\mathrm{CNPq}$ and CAPES.

\section{References}

[1] Hunt KH. Kinematic geometry of mechanisms. vol. 7 of The Oxford engineering science series. Oxford: Clarendon; 1990. Reprinted with corrections [from the 1978 edition].

[2] Gosselin CM, Angeles J. Singularity analysis of closed-loop kinematic chains. IEEE Transactions on Robotics and Automation. 1990 Jun;6(3):281290. Available from: http://ieeexplore.ieee.org/ xpls/abs_all.jsp?arnumber $=56660$.

[3] Zlatanov D, Bonev IA, Gosselin CM. Constraint singularities of parallel mechanisms. In: IEEE International Conference on Robotics and Automation. ICRA '02. vol. 1. Washington; 2002. p. 496502. Available from: http://ieeexplore.ieee.org/ xpls/abs_all.jsp?arnumber $=1013408$.

[4] Davies TH. Dual coupling networks. Proceedings of the Institution of Mechanical Engineers, Part C: Journal of Mechanical Engineering Science. 2006;220(8):1237-1247. Available from: https://dspace.Iboro.ac.uk/dspace-jspui/ handle/2134/4667.

[5] Davies TH. Simple examples of dual coupling networks. In: Merlet JP, Dahan M, editors. Proceedings of Twelfth World Congress in Mechanism and Machine Science. Besançon, France: IFToMM; 2007. Available from: https://dspace. Iboro.ac.uk/ dspace-jspui/handle/2134/3984.

[6] Davies TH. The 1887 committee meets again. Subject: freedom and constraint. In: Ball 2000 Conference, University of Cambridge, Cambridge University Press, Trinity College Proceedings of a Symposium commemorating the Legacy, Works, and Life 
of Sir Robert Stawell Ball upon the $100^{\text {th }}$ Anniversary of A Treatise on the Theory of Screws. Trinity College: University of Cambridge; 2000. p. 156. Available from: https://dspace.1boro.ac.uk/ dspace-jspui/handle/2134/700.

[7] Davies TH. Freedom and constraint in coupling networks. Proceedings of the Institution of Mechanical Engineers, Part C: Journal of Mechanical Engineering Science. 2006;220(7):989 1010. Available from: https://dspace.1boro.ac. uk/dspace-jspui/handle/2134/5892.

[8] Shai O, Pennock GR. Extension of graph theory to the duality between static systems and mechanisms. Journal of Mechanical Design. 2006;128(1):179-191. Available from: http: //mechanicaldesign. asmedigitalcollection. asme.org/article.aspx?articleid=1448813.

[9] Gosselin F, Lallemand JP. A new insight into the duality between serial and parallel non-redundant and redundant manipulators. Robotica. 2001;19(04):365-370. Available from: http://journals.cambridge.org/production/ action/cjoGetFulltext?fulltextid=80022.

[10] Huang C. Some application of the principle of duality to mechanism analysis. Journal of the Chinese Society of Mechanical Engineers. 1994;15:283-283.

[11] Bruyninckx H. Kinematically dual manipulators. In: 1999 IEEE International Conference on Robotics and Automation. vol. 2; 1999. p. 11941199. Available from: http://ieeexplore.ieee. org/xpls/abs_all.jsp?arnumber $=772524$.

[12] Shai O. The duality relation between mechanisms and trusses. Mechanism and Machine Theory. 2001;36:343369. Available from: http://www.sciencedirect. $\mathrm{com} / \mathrm{science/article/pii/S0094114X00000501.}$

[13] Shai O. Utilization of the dualism between determinate trusses and mechanisms. Mechanism and Machine Theory. 2002;37:1307-1323. Available from: http://www.sciencedirect.com/science/ article/pii/S0094114X02000381.

[14] Ruiz López I, Rico Martínez JM, Cervantes Sánchez JJ, Gallardo J, Orozco H. The duality relation between mechanisms and structures: a different view. ASME 2006 International Design Engineering Technical Conferences and Computers and Information in Engineering Conference (IDETC/CIE2006). 2006;2006(42568):1131-1145. Available from: http://proceedings . asmedigitalcollection . asme . org/proceeding . aspx?articleid=1595119.

[15] Davies TH. Couplings, coupling networks and their graphs. Mechanism and Machine Theory. 1995;30(7):991-1000. Graphs and Mechanics First International Conference, Zakopane, Poland, 1993. Available from: https://dspace.1boro.ac. uk/dspace-jspui/handle/2134/9048.
[16] Shai O, Pennock GR. A study of the duality between planar kinematics and statics. Journal of Mechanical Design. 2006;128(3):587-598. Available from: http: //mechanicaldesign. asmedigitalcollection. asme.org/article. aspx?articleid=1448948.

[17] Hunt KH. Special configurations of robot arms via screw theory - Part 1. Robotica. 1986 Jul;4(3):171-179. Available from: http://journals. cambridge.org/action/ displayAbstract? fromPage=online\&aid $=4462876$.

[18] Hunt KH. Robot kinematics - A compact analytic inverse solution for velocities. Journal of Mechanisms Transmissions and Automation in Design. 1987 March;109:42-49. Available from: http: //mechanicaldesign. asmedigitalcollection. asme.org/article.aspx?articleid=1452875.

[19] Davies TH. Mechanical networks - I Passivity and redundancy. Mechanism and Machine Theory. 1983;18(2):95-101. Available from: http://www.sciencedirect. com/science/article/B6V46-482GNP1-60/2/ 99ec936b4d627c7dbc29fbd976b5e2db.

[20] Zlatanov D, Fenton RG, Benhabib B. Singularity analysis of mechanisms and robots via a motion-space model of the instantaneous kinematics. In: Straub E, Sipple RS, editors. IEEE International Conference on Robotics and Automation. vol. 2. Los Alamitos, CA, USA: IEEE Computer Society; 1994. p. 980-985. Available from: http://ieeexplore.ieee.org/xpl/ articleDetails.jsp?arnumber $=351326$.

[21] Shearer JL, Murphy AT, Richardson HH. Introduction to system dynamics. Addison-Wesley Series in Systems and Controls. Reading: Addison-Wesley; 1967.

\section{Appendix}

\section{Notation}

$a$ the rank of the network unit action matrix, $\hat{\mathbf{A}}_{N}$ $\left[\hat{\mathbf{A}}_{D}\right]_{d \times C}$ unit action matrix of the direct couplings

$\left[\hat{\mathbf{A}}_{N}\right]_{d k \times C}$ network unit action matrix of a coupling network

$\mathbf{B}_{l \times e} \quad$ fundamental circuit matrix of a coupling graph $G_{C}$

$\left[\mathbf{B}_{M}\right]_{l \times F} \quad$ fundamental circuit matrix of a motion graph $G_{M}$

$\left[\mathbf{B}_{i}\right]_{F \times F} \quad$ diagonal matrices from row $i$ of $\mathbf{B}_{M}$

c gross degree of constraint (DOC) of a direct coupling

$C \quad$ gross DOC of a coupling network $=\sum c$

$C_{N} \quad$ nett DOC of a coupling network

$d \quad$ minimum order of the screw system, $1 \leq d \leq 6$

$e \quad$ number of couplings in a coupling network and edges of a coupling graph 
$f \quad$ gross degree of freedom (DOF) of a direct coupling

$F \quad$ gross DOF of a coupling network $=\sum f$

$F_{N} \quad$ nett DOF of a coupling network

$G_{A} \quad$ action graph of a coupling network

$G_{C} \quad$ coupling graph of a coupling network

$G_{M} \quad$ motion graph of a coupling network

$\mathbf{J}_{C} \quad$ matrix created from the cofactors of the Jacobian

$k \quad$ number of independent cutsets of a coupling graph $G_{C}$

$l \quad$ number of independent circuits (loops) of a coupling graph $G_{C}$

$m$ the rank of the network unit motion matrix, $\hat{\mathbf{M}}_{N}$

$\left[\hat{\mathbf{M}}_{D}\right]_{d \times F}$ unit motion matrix of the direct couplings of a coupling network

$\left[\hat{\mathbf{M}}_{N}\right]_{d l \times F}$ network unit motion matrix of a coupling network

$\Psi_{C} \quad$ vector of magnitudes of action screws

$\psi_{F} \quad$ vector of magnitudes of motion screws

$\mathbf{Q}_{k \times e} \quad$ fundamental cutset matrix of a coupling graph $G_{C}$

$\left[\mathbf{Q}_{A}\right]_{k \times C} \quad$ fundamental cutset matrix of an action graph $G_{A}$

$\{r, s, t\} \quad$ angular velocity components parallel with the $x$-, $y$ - and $z$-axes, respectively

$\{u, v, w\} \quad$ velocity components along the $x-, y$ - and $z$ axes, respectively, of the point on the body, or an imaginary extension of the body, located at the origin, caused by angular or translational velocity

$\{R, S, T\}$ moment components along the $x$-, $y$ - and $z$ axes, respectively, at the origin, caused by a force or torque

$\{U, V, W\}$ force components parallel with the $x-, y$ - and $z$-axes, respectively 